\title{
Identification of iron by methods of cytochemical analysis in the spleen tissues during experimental radiation pathology
}

\author{
N.V. Baymatov ${ }^{1, *}$, R.R. Farkhutdinov ${ }^{2}, V . N . \mathrm{Kozlov}^{3}$, V.N. Baymatov ${ }^{4}$, and A.N. Kryachko ${ }^{5}$ \\ ${ }^{1}$ Federal Medical and Biological Agency of the Russian Federation, Medical and Sanitary Unit No. \\ 93, Moscow, Russian Federation \\ ${ }^{2}$ FSBEI HE "Bashkir State Medical University" Ufa, Russian Federation \\ ${ }^{3}$ Bashkir Institute of Technology and Management (branch) Federal State Budget Educational \\ Institution of Higher Education «K.G. Razumovsky Moscow State University of technologies and \\ management (the First Cossack University)», Meleuz, Russia Federation \\ ${ }^{4}$ Moscow State Academy of Veterinary Medicine and Biotechnology. K.I. Scriabin, Moscow, Russian \\ Federation \\ ${ }^{5}$ Erakond LLC, Sterlitamak, Russian Federation
}

\begin{abstract}
The article presents the results of studying the radio protective properties of alfalfa extract (dietary supplement "Erakond"). A decrease in the iron content in macrophages of the red pulp of the spleen was found experimentally using models of experimental radiation pathology induced by $\mathrm{y}$-irradiation of white rats in a single dose of $4 \mathrm{~Gy}$ at a dose rate of 1.7 Gy/min. The mechanisms of pathological processes in acute radiation pathology, leading to a decrease in the content of endogenous iron at the systemic, organ, cellular and subcellular levels, have been disclosed. Violation of the processes of enteral absorption of minerals as a result of pronounced destructive-inflammatory processes in the intestinal wall, damage to the hematopoiesis-inducing environment in the red bone marrow, inhibition of cytokine synthesis (IL-1, IL-3, IL-6, IL-7) and granulocytic macrophage colony-stimulating factor (GM-CSF) reduce the level of deposited iron in acute radiation pathology. In rats treated with 40 $\%$ "Erakond" aqueous extract before and after irradiation, there is a relatively high level of deposited iron in the spleen tissues in comparison with animals of the comparison group, which were on a general food ration.
\end{abstract}

\section{Introduction}

In acute and chronic radiation pathology, hypoenergetic conditions are developing as a result of impaired $\mathrm{O}_{2}$ transport and damage to mitochondrial membranes [1, 2, 3]. A true hematopoietic element that plays an important role in the normalization of blood composition is iron, which performs a wide range of functions: it is part of oxidative

\footnotetext{
*Corresponding author: baymatovvaleriy@rambler.ru
} 
enzymes - peroxidase, cytochrome, cytochrome oxidase, thyroperoxidase and myeloperoxidase [4]. Iron is found in more than 70 enzymes and proteins of various functions: in particular, being a part of cytochrome P-450, it neutralizes substances heterogenous to the body, is the most important part of non-porphyrin enzymes - aldolase, aconitase and succinate dehydrogenase. Iron in the form of an iron-sulfur cluster [4Fe-4S] is part of the enzyme aconitase, which catalyzes the stereospecific reaction of citrate isomeration to isocitrate. The catalytic activity of aconitase depends on the specific conformation, for which the iron-sulfur cluster [4Fe-4S] and several amino acid residues are responsible. The iron content is regulated mainly by the intensity of its absorption in the intestine. In the cells of the intestinal mucosa, iron in the trivalent form $\mathrm{Fe}^{3+}$ combines with apoferritin to form ferritin. Further iron transport in vivo is carried out in the form of a complex with $\beta$-globulins of blood serum - the complex is called transferin [5]. It was established that great trefoil extract - Erakond dietary supplement [6] is an effective ironcontaining radioprotector with pronounced antioxidant properties.

The aim of the research was to evaluate the effect of great trefoil extract on the iron content in organs of white rats exposed to radiation in the experiment.

Materials and methods. The experiments were carried out on 48 laboratory outbred white male Wistar rats of mature age weighing 180-220 g. The selection of animals into groups was carried out according to the principle of analogues considering age, live weight, fatness, origin, breed. During the experiments, the provisions of the International Declaration on the humane animal treatment were fully observed.

Experimental animals were divided into 4 groups of 12 in each: the first one was biological control, animals of the $2 \mathrm{nd}$, 3rd and 4th groups were irradiated once at the Republican Oncological Dispensary (Ufa) at the SL-75-5-M radiotherapy unit at a dose of 4 Gy at a dose rate of $1.7 \mathrm{~Gy} / \mathrm{min}$. Animals of the 3rd group were on the standard vivarium diet for a month. During 10 days before and after irradiation, rats of the 4th group received a $40 \%$ solution of "Erakond" daily with feed at the rate of $7-10 \mathrm{ml} / \mathrm{kg}$ of body weight. At the end of the experiment, 30 days after irradiation, the animals were removed from the experiment by one-stage decapitation under ether anesthesia, followed by the removal of internal organs for histological studies. The content of endogenous iron in spleen tissues was determined by the Perls method.

Research results. Cytochemical reaction to iron in animals' spleen: in the control group of rats, an intense reaction to iron is determined in macrophages located in the red pulp of the spleen, while iron is not detected in the white pulp (Fig. 1). Iron granules in the cytoplasm of macrophages are evenly distributed; however, individual macrophages are characterized by large iron granules. The red pulp is represented by sinuses and cords of cells containing macrophages, erythrocytes, platelets, granulocytes, lymphocytes and plasma cells. White pulp is a combination of lymphoid spleen tissue represented by accumulations of T-lymphocytes around the arteries. 


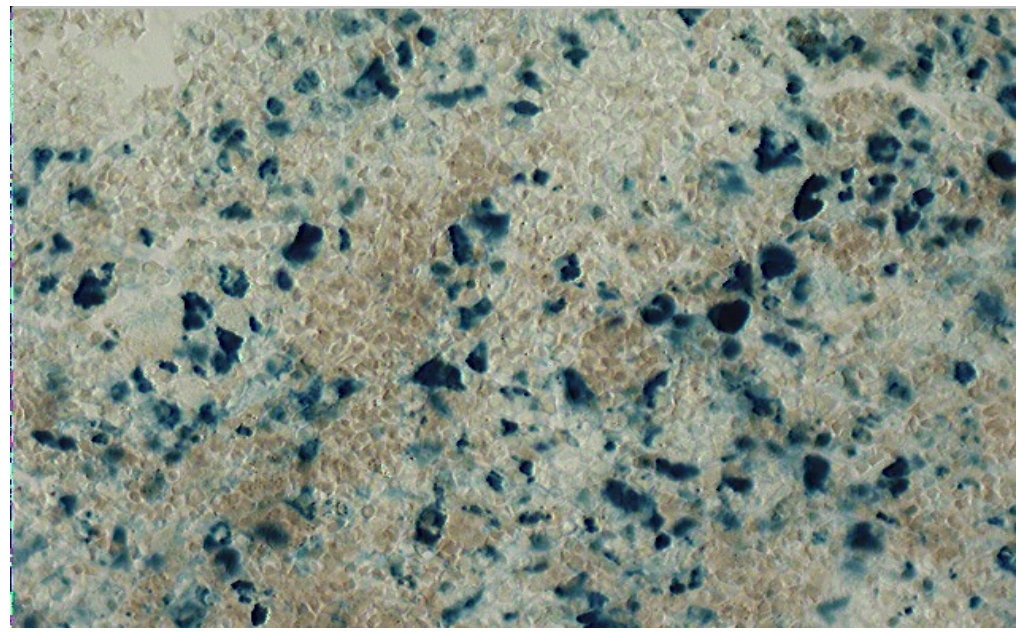

Fig. 1. High reaction to iron in spleen macrophages in animals of the control group. Perls reaction. Photomicrography. cir. 10, vol. 40.

In the human body, the liver and spleen collectively deposit $\approx 25 \mathrm{mg}$ of iron, which is released during the constant erythrocytes' decay. Iron in ferritin is in oxidized form in the composition of an inorganic iron-containing compound, and the chains of the inorganic polymer $\mathrm{O}=\mathrm{Fe}-\mathrm{OH} . . . \mathrm{O}=\mathrm{Fe}-\mathrm{OH}$ are located between the peptide chains of the protein part apoferritin. Macrophages phagocytize debris of tissue matrix and cells. Iron deposited in macrophages is again used in the biosynthesis of heme-containing enzymes and hemoglobin. The activity of macrophages predetermines the outcome of inflammatory diseases, oncopathology and radiation pathology: an activated macrophage releases more than 60 factors that ensure both the elimination of damaged structures and proliferation.

In experimental animals of the 2nd group irradiated at $4 \mathrm{~Gy}$ dose, the functions of hematopoiesis and destruction of obsolete or damaged erythrocytes and platelets are suppressed in the spleen. In general, this is manifested by a decrease in the histochemical reaction for iron in the red pulp of the spleen (Fig. 2).

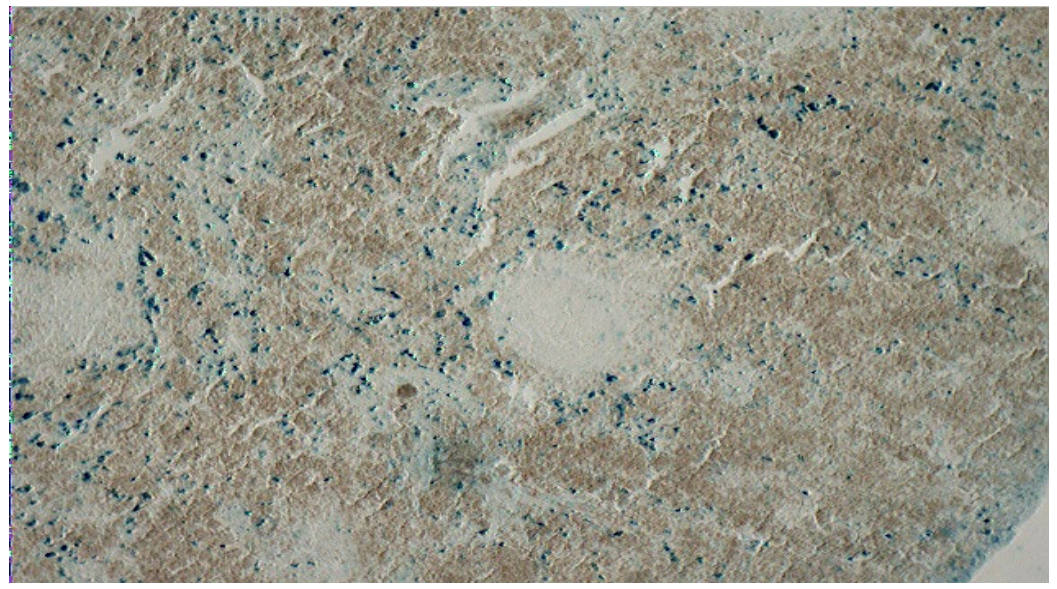

Fig. 2. Decrease in the reaction intensity to iron in macrophages of the red spleen pulp with $\gamma^{-}$ irradiation of animals at 4 Gy dose (group 2). Perls reaction. Photomicrography. cir. 10, vol. 20. 
Macrophages are functionally inactive; iron granules in the cytoplasm of macrophages are small and evenly distributed in the cytoplasm, however, there are individual macrophages of the red spleen pulp with an intense reaction to iron. The histograms of the spleen in rats of the 2nd and 3rd groups did not differ from each other in the severity of the cytochemical reaction to iron. The decrease in the content of deposited iron in the studied organs is apparently due to impaired absorption of $\mathrm{Fe}^{2+}$ by epithelial cells of the intestinal mucosa, i.e., the processes of apoferritin formation are blocked.

In animals that received Erakond in addition to the diet (group 4), a significant increase in the histochemical reaction intensity for iron in the spleen was determined (Fig. 3). Macrophages containing iron granules are evenly distributed in the red spleen pulp and the amount of iron granules is significantly

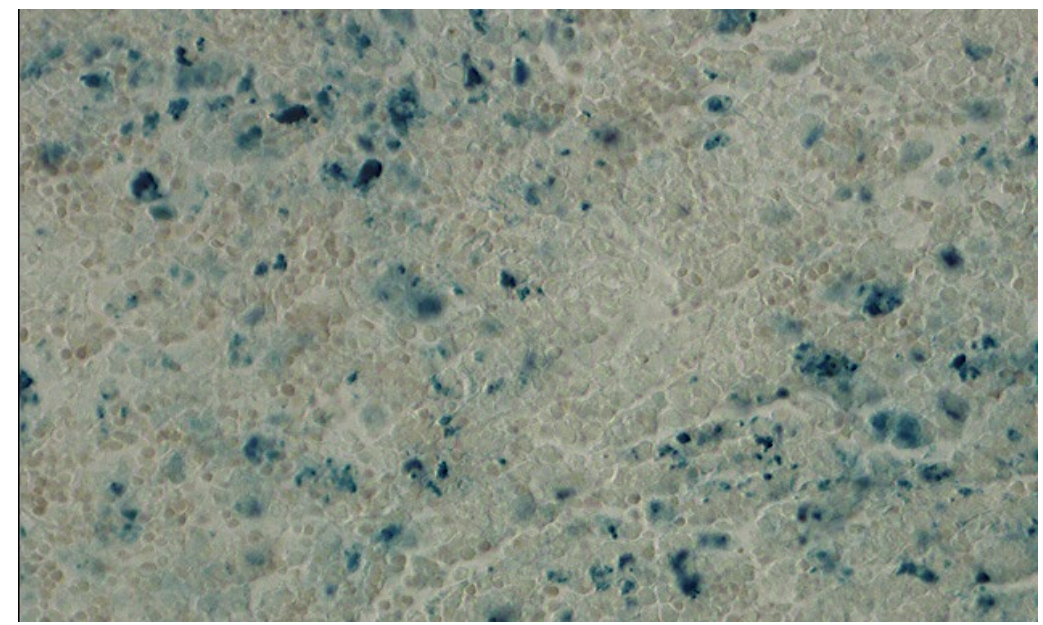

Fig. 3. Macrophages of the red spleen pulp of animals treated with Erakond before and after $\gamma$ irradiation. Perls reaction (4th group). Photomicrography. cir. 10, vol. 40

less relative to animals of the control group. Individual macrophages contain a large amount of iron granules. It is also characteristic that in the marginal sinus and sometimes in the center of the lymph node of the white pulp, macrophages with a positive reaction to iron are detected. The results of cytochemical studies showed that the introduction of a radioprotectant into the diet of the 4th group of animals promoted an increase in the activity of the nutrients deposition processes in hepatocytes and spleen.

Inhibition of erythropoiesis as a result of radiation exposure is one of the factors that predetermine a decrease in the content of deposited iron in tissues. The ancestor of all formed blood cells is the hematopoietic stem cell (pPSC). Precursors of formed blood cells are capable of reproduction only in the presence of stimulating factors - cytokines produced by monocytes, macrophages, lymphocytes and stromal elements of the bone marrow. The proliferation of pPSCs (stem cells) is influenced by a class of cytokines called "interleukins", in particular interleukin-1 (IL-1) and interleukin-3 (IL-3). Interleukin-1 is a cytokine, a mediator of inflammation and immunity, which is synthesized by activated macrophages, B-lymphocytes and fibroblasts. Inhibition of bone marrow synthesis of Blymphocytes in acute radiation pathology is accompanied by interleukin-1 synthesis inhibition, which stimulates the processes of erythro-, leukopoiesis, and affects the processes of heat production. IL-1 macrophages stimulate the activity of both Tlymphocytes and B-lymphocytes. Hematopoiesis is induced with the participation of interleukin-3, which controls the proliferation processes of leukocytes, erythrocytes and 
eosinophils. IL-1, IL-3 and IL-6 belong to the so-called early-acting hematopoietic factors of macrophages, stromal, adipose and endothelial cells. The main substance of the connective tissue of the bone marrow is the physiologically active environment that allows to consider it as the most important regulator of hematopoiesis. The late-acting factors of hematopoiesis include granulocyte-macrophage colony-stimulating factor (GM-CSF). GMCSF is a high molecular weight peptide compound that, in combination with erythropoietin (EPO), is involved in erythrocyte differentiation. Fibroblasts - red brain cells capable of secreting IL-3 and IL-7 - take part in the processes of bone marrow hematopoiesis. In addition, fibroblasts synthesizing components of the extracellular matrix (collagen, elastin, fibronectin, glycosaminoglycans, proteoglycans) produce granulocyte colony-stimulating factor (G-CSF) and macrophage colony-stimulating factor (M-CSF). Thus, damage to the so-called hematopoiesis-inducing environment in the red bone marrow when exposed to ionizing radiation is one of the factors that inhibit erythropoiesis [7].

\section{Conclusions}

Violation of iron absorption processes in acute radiation pathology is the result of the phenomena of necrosis, hyperplasia, infiltration of the entire intestinal mucosa. As a result of own research, it was found that a pronounced inflammatory process is observed under the influence of $\gamma$-irradiation in the small intestine, spreading to all layers of the mucous membrane [8]. With the observed destructive-dystrophic processes, the mechanisms of impaired iron absorption develop in the following sequence: acute radiation pathology $\rightarrow$ destructive-inflammatory processes in the intestinal wall $\rightarrow$ decrease in $\mathrm{pH} \rightarrow$ violation of enteral absorption processes of iron $\rightarrow$ inhibition of transferrin activity $\rightarrow$ anemia $\rightarrow$ decrease in the level of deposited iron. The preventive measures taken before and after irradiation of animals contributed to an increase in the metabolic status and acceleration of the recovery processes in the studied organs after their damage by radiation.

\section{References}

1. I.A. Nikitina, Problems of health and ecology 1(27), 102-106 (2011)

2. I.M. Obodovsky, Fundamentals of radiation and chemical safety, 304 (2013)

3. V.G. Petin, Radiation and risk, 12, 117-134 (2001)

4. B.A. Frolov, Physiology and pathology of neuroendocrine regulation, 320 (2006)

5. A.I. Suslova, Innovative technologies in pharmacy: Collection of scientific papers, 483-488 (2019)

6. V.N. Baimatov, Journal of Engineering and Applied Sciences, 13(8), 6576-6583 (2018)

7. N. Ahluwalia, Am. J. Clin. Nutr, 79, 516-521 (2004)

8. N.V. Baymatov, Morphology, 157(2-3), 26 (2020) 